\title{
BMJ Open Association of Meniere's disease and retinal vascular calibre: a prospective observational study in China
}

\author{
Yixu Wang, ${ }^{1}$ Tongxiang Diao, ${ }^{1}$ Lin Han, ${ }^{1}$ Ye Tao, ${ }^{2}$ Lisheng Yu ${ }^{1}$
}

To cite: Wang Y, Diao T, Han L, et al. Association of Meniere's disease and retinal vascular calibre: a prospective observational study in China. BMJ Open 2018;8:e022069. doi:10.1136/ bmjopen-2018-022069

- Prepublication history for this paper is available online. To view these files please visit the journal online (http://dx.doi. org/10.1136/bmjopen-2018022069).

Received 1 February 2018 Revised 27 August 2018 Accepted 7 September 2018

Check for updates

(C) Author(s) (or their employer(s)) 2018. Re-use permitted under CC BY-NC. No commercial re-use. See rights and permissions. Published by BMJ.

${ }^{1}$ Department of Otolaryngology, Head and Neck Surgery, People's Hospital, Peking University, Beijing, China ${ }^{2}$ Department of Ophthalmology, People's Hospital, Peking University, Beijing, China

Correspondence to

Dr Lisheng Yu;

yixu2007@163.com

\section{ABSTRACT}

Objective It is believed that Meniere's disease (MD) is associated with vascular disorders, but few studies have reported the relationship between retinal vascular disorders and MD. We evaluated and compared the retinal vascular calibres in patients with MD with healthy subjects matched for age, sex and vascular risk factors using retinal photographs to explore the association between MD and retinal vascular calibre.

Study design A prospective study.

Setting Tertiary referral centre.

Participants Sixty patients with MD and 62 healthy subjects matched for age, sex and vascular risk factors were enrolled in this study. Twenty-four patients with MD had migraines, and 36 patients with MD did not have migraines.

Main outcome measure Retinal vascular calibres were calculated and compared not only between patients with MD and healthy subjects but also between subgroups of patients with MD.

Results Compared with healthy subjects, patients with MD had a slightly larger retinal artery calibre (126.30 $\pm 10.45 v s 119.61 \pm 15.86, p=0.006)$ and $a$ higher retinal artery/vein ratio $(0.79 \pm 0.09 v s \quad 0.75 \pm 0.10$, $\mathrm{p}=0.005)$. Among patients with MD, those with migraines had a larger retinal artery calibre $(130.73 \pm 11.55 \mathrm{vs}$ $123.35 \pm 8.61, p=0.006$ ) than those without migraines. Moreover, the presence of migraines and the high frequency of vertigo attacks appeared to increase the retinal artery calibre.

Conclusions Our study suggests that a relationship exists between retinal vascular calibre and MD. Although the pathophysiological relationship between migraine and MD remains unclear, the presence of migraine attacks may aggravate endolymphatic hydrops (EH) and accelerate the outflow of EH in patients with MD. More extensive studies are required to explore the association between retinal vascular calibre and MD.

\section{INTRODUCTION}

Since Meniere's disease (MD) was described by Prosper Meniere in 1861, it has been regarded as a chronic illness that affects a large number of patients worldwide every year. ${ }^{1}$ Studies conducted in Finland, Italy, UK and the USA have estimated the prevalence of $\mathrm{MD}$ to be $0.043 \%, 0.086 \%, 0.51 \%$ and $0.22 \%$, respectively. ${ }^{2-6} \mathrm{MD}$ is characterised by
Strengths and limitations of this study

The first prospective study to explore the association between Meniere's disease (MD) and retinal vascular calibre in China.

- The healthy controls were matched for sex, age and vascular risk factors to potentially reduce the effect of confounding factors.

- There is the potential for recall bias as MD and migraine were always diagnosed based on clinical symptoms.

- Single-centre study that included a limited number of patients.

paroxysmal vertigo lasting from minutes to hours, accompanied by fluctuating hearing loss, tinnitus and aural pressure. Although many studies have proven that the primary histopathological substrate is endolymphatic hydrops $(\mathrm{EH})$, the real pathological mechanism remains unclear. ${ }^{7}$ At present, it is believed that MD is a disease of unknown aetiology with multiple contributing factors, including family history, viral infection, allergy and autoimmunity. EH is considered a necessary but insufficient factor for the progression of MD. ${ }^{8}$ Though some studies suggest that the occurrence and progression of $\mathrm{MD}$ is closely related to vascular disorders, these studies are relatively few. ${ }^{7}$

Large studies have reported that MD can coexist with migraines and suggest that there is a close pathophysiological relationship between these two disorders. ${ }^{9}{ }^{10}$ Migraines occur more commonly in patients with MD than in the general population, which seems to support the concept that the disorders share a common pathophysiological process. ${ }^{11}$ In fact, several scholars have regarded migraine as an additional symptom in typical MD attacks. Although the pathogenesis of migraine is still debated, there seems to be an association with the neurovascular system. ${ }^{12}$ Retinal vascular disorder has been reported in patients with migraine 
and is thought to result from activation of the trigeminal vascular system (TVS), which innervates the blood vessels of the eyes. ${ }^{13}$ It has been demonstrated that the inner ear blood flow is also directly affected by the trigeminal nerve through innervation of cochlear vasculature. ${ }^{14-16}$ We therefore hypothesised that retinal vascular disorders may exist in patients with MD due to the close relationship between MD and migraine.

The retina has been regarded as a unique site where the microvasculature can be visualised and observed directly in vivo. ${ }^{17}$ Retinal photographic imaging techniques can accurately measure and quantify subtle abnormalities and changes in the retinal microvasculature. Several researchers have previously reported that branch retinal artery occlusion and retinal perivasculitis occur in patients with MD. ${ }^{18} 19$ In this study, we examined our hypothesis and the association between MD and retinal vascular calibre. The aim of our study was (1) to evaluate and compare retinal vascular calibres between patients with MD and healthy subjects matched for age, sex and vascular risk factors using retinal photographs and (2) to compare the clinical characteristics and retinal vascular calibres between patients with MD alone and those with migraines.

\section{METHODS}

\section{Study design}

We conducted a prospective, observational study. Based on the presence of migraine, studied patients with MD were divided into two groups: patients with MD alone and those with migraine. The diagnosis of MD and migraine was made on the basis of the criteria defined by the American Academy of Otolaryngology-Head and Neck Surgery (AAO-HNS) and International Headache Society (IHS). ${ }^{20}{ }^{21}$ The study contained two parts. (1) We evaluated and compared retinal vascular calibres between patients with MD alone and healthy subjects matched for age, sex and vascular factors to determine whether there was an association between MD and retinal vascular calibres. (2) We compared the clinical characteristics and retinal vascular calibres between patients with MD alone and those with migraine.

\section{Patients}

Studied patients were enrolled in the Otolaryngologic Department in Peking University People's Hospital between 1 June 2014 and 1 January 2016. Patients aged 20-70 years old were eligible for enrolment if they complained mainly about two or more spontaneous episodes of vertigo lasting from $20 \mathrm{~min}$ to 12 hours within the past several years. Those vertigo patients who were diagnosed with MD according to AAO-HNS (box 1) were included in this study. ${ }^{20}$ All patients underwent many imaging examinations such as CT and MRI to exclude acoustic neuroma and to make a diagnosis of EH. The control group consisted of 62 healthy subjects matched for age, sex and vascular risk factors. These healthy subjects
Box 1 Diagnosis of Meniere's disease

Certain Meniere's disease
Definite Meniere's disease, plus histopathological confirmation
Definite Meniere's disease
Ionger or more definitive spontaneous episodes of vertigo 20 min or
Audiometrically documented hearing loss on at least one occasion
- Tinnitus or aural fullness in the treated ear
Other causes excluded
Probable Meniere's disease
One definitive episode of vertigo
- Audiometrically documented hearing loss on at least one occasion
Tinnitus or aural fullness in the treated ear
Other causes excluded
Possible Meniere's disease
Iosisodic vertigo of the Meniere type without documented hearing
- Sensorineural hearing loss, fluctuating or fixed, with dysequilibrium
but without definitive episodes
Other causes excluded

had no complaints of symptoms of hearing loss or vertigo attacks and had not been previously diagnosed with either MD or migraine. Key exclusion criteria included having a history of ocular surgery influencing the examination of the fundus, current or a history of hypertension and diabetes that affected the retinal vascular calibre, current ocular inflammation, glaucoma or other diseases that could alter the calibre of blood vessels, autoimmune diseases, smoking history and ischaemic heart disease. We also excluded patients who had other peripheral vertigo such as vestibular migraine (VM) alone, benign paroxysmal positional vertigo, central vertigo, psychogenic vertigo and vertigo caused by systemic disease.

Because of the similarities in the clinical features between MD and VM and because there are no examinations available to adequately distinguish the two disorders, many studies have suggested that a history of progressive hearing loss is sensitive and specific for MD. Ultimately, we chose to regard the symptom of progressive hearing loss as a criteria for separating MD and VM.

Several professors skilled in treating vertigo reviewed our data and concluded that our study diagnoses were based on the AAO-HNS definition of MD, third edition of the International Classification of Headache Disorders (ICHD-III) criteria for migraine and VM. ${ }^{20}{ }^{21}$ These diagnoses were not mutually exclusive, and patients could be diagnosed with MD with migraine or MD with VM when they met the diagnostic criteria for both diseases. We put MD with VM in the category of MD with migraine because $\mathrm{VM}$ is a special type of migraine according to ICHD-III. ${ }^{21}$ The study was approved by the hospital's ethics committee. All the patients provided written informed consent.

We collected detailed information about demographic and clinical characteristics including age and sex, course of $\mathrm{MD}$, duration and frequency of vertigo attacks, 


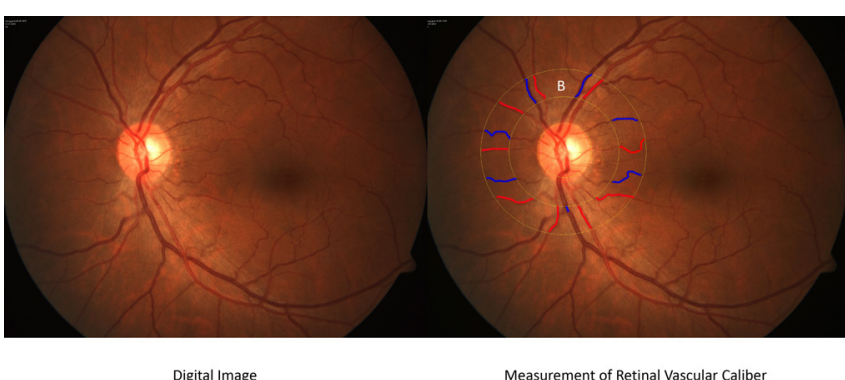

Figure 1 Representative image showing retinal vascular calibre measurement used in this study. Zone B: 0.5-1.0 disc diameters from the disc margin. Red line: artery tracking. Blue line: vein tracking.

tinnitus and aural fullness (yes or no), photophobia and phonophobia (yes or no) and visual aura (yes or no). Some studies reported a close association between retinal vascular calibres and vascular risk factors such as systolic pressure, diastolic pressure, blood glucose, serum triglycerides, cholesterol, high-density lipoprotein cholesterol and low-density lipoprotein cholesterol levels. We measured and matched these risk factors between studied patients and healthy controls to decrease the impact of these confounding variables on retinal vascular calibres. In addition, pure tone hearing tests of all patients had been performed many times in the past.

\section{Measurement of retinal vascular calibre}

Retinal photography under cycloplegia was performed on all patients with MD and control subjects. Retinal colour images (resolution $2048 \times 2048$ pixels) were acquired at a $30^{\circ}$ angle with the optic nerve head centred by using the Canon CX-1 Fundus camera (Canon, Japan). The measurement of retinal vascular calibres was taken on disc-centred photographs with the use of computer-assisted software (Canon, Japan). Artery:vein ratio (AVR), central retinal artery equivalent (CRAE) and central retinal vein equivalent (CRVE) were obtained by using arterial and venous diameters. Arteries and veins located within the area of one-half disc diameter (DD) to one full DD from the optic disc margin were selected for measurements (AVR, CRAE and CRVE) (figure 1). These measurements were used for describing the physical structure of different retinal arteries and veins such as luminal calibre. The detailed definitions and measurement methods for each retinal vascular parameter are previously explained elsewhere. ${ }^{22} 23$ In a past study, we compared the retinal vascular calibre between both eyes of patients with unilateral MD and found that the retinal vascular calibre on the side of the affected ear was significantly larger than that on the contralateral side. Therefore, in this study, all vessels were measured in the eye that was on the same side as the affected ear in patients with unilateral MD, but the vessels of the eye on the same side of the more seriously affected ear were measured in patients with bilateral MD.

\section{Patient and public involvement}

No patients were involved in the development and design of the study.

\section{Statistical analysis}

SPSS software (V.21.0, SPSS) was used for statistical analyses. For categorical data, frequencies and percentages were calculated to describe the distributions of subgroups among patients according to sex, disease course, duration and frequency of vertigo attacks, photophobia, phonophobia and visual aura. Categorical data were compared using $\chi^{2}$ or Fisher exact tests if appropriate. Means $( \pm \mathrm{SD})$ were used to summarise the average levels of quantitative data such as age, body mass index, retinal vascular calibres and vascular risk factors. A Shapiro-Wilk test was used for examining whether the measured values were in accordance with a normal distribution. At eye measurement level, the data of retinal vascular calibres that were distributed normally were compared by two-sample t-test between patients with MD and healthy controls, and between patients with MD alone and those with migraine.

Generalised linear regression analysis, in which age, sex, duration of MD, frequency of vertigo attacks and migraine symptoms were included as covariates, was used to assess the correlation of these independent factors with the retinal calibres among patients with MD. The linear regression analysis contained two models, an unadjusted analysis model and an adjusted analysis model that was adjusted for age and sex. Beta coefficient and 95\% CI were calculated according to model-variable coefficients and SE, respectively. All $\mathrm{p}$ values of less than 0.05 were considered to indicate statistical significance.

Table 1 Demographic characteristics and vascular risk factor levels in studied patients with MD and healthy controls

\begin{tabular}{llll}
\hline & MD patients & $\begin{array}{l}\text { Healthy } \\
\text { controls }\end{array}$ & P values \\
\hline Age (years) & $53.58 \pm 10.74$ & $53.32 \pm 10.46$ & 0.89 \\
\hline Women (\%) & $31(51.7)$ & $32(51.6)$ & 0.85 \\
\hline BMI (scores) & $24.37 \pm 3.43$ & $24.54 \pm 3.45$ & 0.78 \\
\hline $\begin{array}{l}\text { Current systolic } \\
\text { pressure (mm Hg) }\end{array}$ & $122.83 \pm 14.05$ & $120.63 \pm 10.66$ & 0.31 \\
$\begin{array}{l}\text { Current diastolic } \\
\text { pressure (mm Hg) }\end{array}$ & $80.18 \pm 8.48$ & $78.74 \pm 7.19$ & 0.29 \\
\hline Blood glucose & $5.77 \pm 1.16$ & $5.60 \pm 0.75$ & 0.31 \\
\hline Triglyceride (mmol/L) & $1.51 \pm 1.20$ & $1.44 \pm 1.25$ & 0.75 \\
\hline Cholesterol (mmol/L) & $4.91 \pm 0.87$ & $4.85 \pm 0.80$ & 0.69 \\
\hline HDL-C (mmol/L) & $1.18 \pm 035$ & $1.17 \pm 0.33$ & 0.54 \\
\hline LDL-C (mmol/L) & $2.97 \pm 0.75$ & $2.94 \pm 0.68$ & 0.87 \\
\hline
\end{tabular}

BMI, body mass index; HDL-C, high-density lipoprotein cholesterol; LDL-C, low-density lipoprotein cholesterol; MD, Meniere's disease. 
Table 2 The clinical characteristics of all 60 patients with MD

\section{MD patients with migraine Patients with MD alone All patients with MD P values}

\begin{tabular}{|c|c|c|c|c|}
\hline \multicolumn{5}{|l|}{ Disease course (no. (\%)) } \\
\hline$<1 / 2$ year & $3(12.5)$ & 8 (22.2) & $11(18.3)$ & 0.340 \\
\hline 1/2-1 year & $2(8.3)$ & $6(16.7)$ & 8 (13.3) & 0.352 \\
\hline$>10$ years & $2(8.3)$ & $4(11.1)$ & $6(10.0)$ & 0.725 \\
\hline \multicolumn{5}{|c|}{ Duration of vertigo attacks (no. (\%)) } \\
\hline $1-6$ hours & $8(33.3)$ & $19(52.8)$ & $27(45.0)$ & 0.138 \\
\hline$>6$ hours & $9(37.5)$ & $3(8.3)$ & $12(20.0)$ & 0.006 \\
\hline \multicolumn{5}{|c|}{ Frequency of vertigo (no. (\%)) } \\
\hline$<1$ week & $10(41.7)$ & $5(13.9)$ & $15(25.0)$ & 0.015 \\
\hline 1 week-1month & $5(20.8)$ & $13(36.1)$ & $18(30.0)$ & 0.206 \\
\hline$>1$ year & $0(0)$ & $2(5.6)$ & $2(3.3)$ & 0.240 \\
\hline Photophobia (no. (\%)) & $10(41.7)$ & $15(41.7)$ & $25(41.7)$ & 0.990 \\
\hline Phonophobia (no. (\%)) & $18(75.0)$ & $9(25.0)$ & $27(45.0)$ & 0.001 \\
\hline Visual aura (no. (\%)) & 7 (29.2) & $3(8.3)$ & $10(16.7)$ & 0.034 \\
\hline Tinnitus (no. (\%)) & $24(82.8)$ & $36(92.3)$ & 60 (88.2) & 0.227 \\
\hline Aura fullness (no. (\%)) & 20 (83.3) & $26(72.2)$ & $46(76.7)$ & 0.319 \\
\hline Stage of MD (no. (\%)) & & & & 0.899 \\
\hline Stage 1 & $2(8.3)$ & $4(10.3)$ & $6(10.0)$ & \\
\hline Stage 2 & $4(16.7)$ & $4(10.3)$ & $8(13.3)$ & \\
\hline
\end{tabular}

Calculated $p$ values were made for comparison between subgroups of patients with MD.

MD, Meniere's disease.

\section{RESULTS}

\section{Demographic characteristics}

In all, 60 patients with MD were enrolled out of 76 subjects (the other 16 patients were excluded from this study based on the exclusion criteria): 31 female patients $(51.7 \%)$ and 29 male patients $(48.3 \%)$. The mean age of the patients with MD and healthy controls was $53.58 \pm 10.74$ and $53.32 \pm 10.46$, respectively. According to the IHS criterion, 24 patients with MD had migraine, and 36 patients with MD did not have migraine. Demographic characteristics and vascular risk factors of patients with MD alone and matched healthy controls are shown in table 1 .

\section{Clinical characteristics}

All patients with MD had vertigo attacks and tinnitus, while most patients with MD had aural fullness (76.7\%). MD patients with migraine had more frequent vertigo attacks than patients with MD alone. The frequency of vertigo attacks in a week was $41.7 \%$ for MD patients with migraine and $13.9 \%$ for MD patients without migraine $(\mathrm{P}=0.015)$. MD patients with migraine experienced a longer duration of vertigo attacks than those without migraine (with an interval of $>6$ hours for $37.5 \% \mathrm{MD}$ patients with migraine and for $8.3 \% \mathrm{MD}$ patients without migraine, $\mathrm{p}=0.006$ ). Compared with patients with $\mathrm{MD}$ alone, more MD patients with migraine complained of symptoms of phonophobia $(75.0 \%$ vs $25.0 \%, \mathrm{p}=0.004)$ and visual aura $(29.2 \%$ vs $8.3 \%, \mathrm{p}=0.034)$ (table 2$)$.

Table 3 Comparison of retinal vascular calibres between patients with MD and matched healthy controls

\begin{tabular}{lccl}
\hline Variable & Matients with & & \\
\hline CRAE $(\mu \mathrm{m})$ & $126.30 \pm 10.45$ & $119.61 \pm 15.86$ & 0.006 \\
CRVE $(\mu \mathrm{m})$ & $159.85 \pm 10.35$ & $161.04 \pm 10.41$ & 0.516 \\
AVR $(\mu \mathrm{m})$ & $0.79 \pm 0.09$ & $0.75 \pm 0.10$ & 0.005 \\
\hline
\end{tabular}

Calculated $p$ values were made for comparison between the group of patients with MD and control group.

AVR, artery:vein ratio; CRAE, central retinal artery equivalent; CRVE, central retinal vein equivalent; MD, Meniere's disease. 
Table 4 Comparison of retinal vascular calibres between subgroups of patients with MD

\begin{tabular}{llccc}
\hline Variable & MD patients with migraine & Patients with MD alone & All patients with MD & P values \\
\hline CRAE $(\mu \mathrm{m})$ & $130.73 \pm 11.55$ & $123.35 \pm 8.61$ & $126.30 \pm 10.45$ & 0.006 \\
CRVE $(\mu \mathrm{m})$ & $160.32 \pm 9.11$ & $159.54 \pm 11.22$ & $159.85 \pm 10.35$ & 0.778 \\
AVR $(\mu \mathrm{m})$ & $0.82 \pm 0.09$ & $0.78 \pm 0.09$ & $0.79 \pm 0.09$ & 0.083 \\
\hline
\end{tabular}

Calculated $p$ values were made for comparison between subgroups of patients with MD.

AVR, artery:vein ratio; CRAE, central retinal artery equivalent; CRVE, central retinal vein equivalent; MD, Meniere's disease.

\section{Eye measurement}

Compared with healthy controls, patients with MD exhibited larger arteries $(126.30 \pm 10.45$ vs $119.61 \pm 15.86$, $\mathrm{p}=0.006)$ and a higher retinal AVR $(0.79 \pm 0.09$ vs $0.75 \pm 0.10$, $\mathrm{p}=0.005$ ) (table 3). For patients with MD, the CRAE was larger in those with migraine than in patients with MD alone $(130.73 \pm 11.55$ vs $123.35 \pm 8.61, \mathrm{p}=0.006)$, but AVR did not differ significantly between subgroups of patients with MD (table 4). The CRVE did not differ significantly either between patients with MD and healthy controls or between subgroups between patients with MD. We set a type I error probability of 0.05 and calculated a power for this two-sample means test of 0.82 (larger than 0.80), which is sufficient for determining the significant difference between patients with MD and controls.

\section{Factors correlated with retinal vascular calibre}

We considered sex, age, frequency of vertigo attacks, symptoms of migraine and duration of vertigo attacks as potential factors correlated with retinal vascular calibre in patients with MD. In our study, age and sex were not significantly correlated with retinal vascular calibre; however, the CRVE was inversely correlated with the duration of vertigo attacks (adjusted beta coefficient, -2.22; $95 \% \mathrm{CI},-7.51$ to $-0.41 ; \mathrm{p}=0.014)$; the CRAE was positively correlated with the presence of migraine (adjusted beta coefficient, $3.19 ; 95 \% \mathrm{CI}, 1.44$ to $6.03 ; \mathrm{p}=0.001$ ) and frequency of vertigo attacks (adjusted beta coefficient, 2.36; $95 \%$ CI, 1.12 to 12.16 ; $\mathrm{p}=0.018$ ) (table 5).

\section{DISCUSSION}

In this prospective study evaluating the association between $\mathrm{MD}$ and retinal vascular calibres, generalised linear regression analysis revealed that the presence of migraine and the frequency of vertigo attacks appear to increase the retinal artery calibre in patients with MD. The results indicated that MD patients with migraine presented with more frequent vertigo attacks, longer duration of vertigo attacks and a larger retinal artery than patients with MD alone. This finding contrasts with those of Gerald $e t a l^{24}$ who reported that the mean retinal arteriolar diameter was slightly smaller in subjects with a history of any migraine, but did not differ significantly from those without a history of any migraine. Our results suggest that screening for the presence of migraine in patients with MD could be based not only on the frequency and duration of vertigo attacks but also on the measurement of retinal artery calibre.

Several studies have demonstrated a relationship between MD and retinal blood vessels. Recupero SM et al described a young patient with MD who was affected by branch retinal artery occlusion and migraine headaches. ${ }^{19}$ Wanger et al presumed that retinal perivasculitis was associated with MD and reported that a common pathogenetic mechanism of microvasculopathy resulted in a parallel clinical course and simultaneous therapeutic response to corticosteroids. ${ }^{18}$ In our analysis of retinal vascular calibre in patients with MD and matched healthy subjects, the CRAE and AVR were larger in patients with MD. Our results suggest there is a close relationship between MD and retinal artery calibre and, furthermore, that the retinal artery calibre could increase in patients with MD.

Some studies have previously reported an association between MD and glaucoma, but the results have always been inconsistent. Setch et al evaluated the audiovestibular function in patients with glaucoma and found a cochlear lesion in these patients. ${ }^{25}$ However, many studies have indicated there was no relationship between MD and glaucoma. Berkowitz et al reported that none of the 10 patients with idiopathic EH had glaucoma. Shapiro et al found no association between glaucoma and hearing loss when evaluating the hearing thresholds of 67 patients with bilateral function. ${ }^{26}$ Kabudwabd et al also showed no evidence of a relationship between glaucoma and inner ear hearing loss, but considered that EH can appear together with glaucoma on the basis of the hypothesis that EH in an early stage may be present without hearing loss. $^{27}$

In our study, 24 of 60 patients with MD (40.0\%) had a history of or current migraine, a finding that is consistent with many scholars who have reported that MD can coexist with migraine as defined by the IHS. ${ }^{9}$ MD patients with migraine had more frequent vertigo attacks than those without migraine (with an interval of $<1$ week, $41.7 \%$ vs $13.9 \%, \mathrm{p}=0.015)$. The incidence of frequent vertigo attacks (with an interval of $<1$ week) in MD patients with migraine was slightly lower than those in the study by Morganti et al, ${ }^{28}$ who reported that $67.6 \%$ of VM patients had more than one incidence of vertigo attacks within a week. Moreover, a greater number of MD patients with migraine had a long duration of vertigo attacks compared with those without migraine $(37.5 \%$ vs $8.3 \%, \mathrm{p}=0.006)$. 


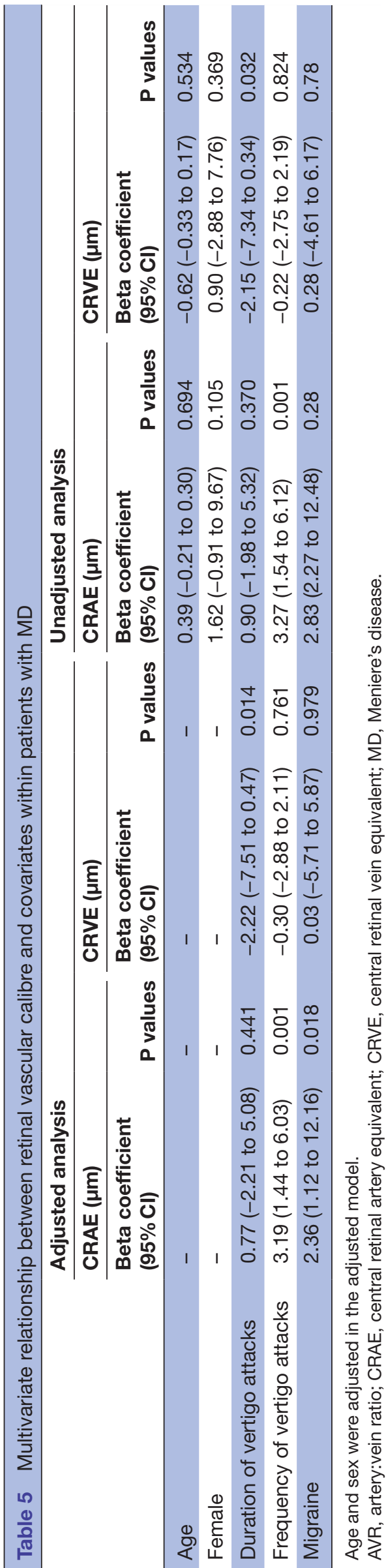

Since MD patients with migraine had more frequent and longer lasting vertigo attacks than those without migraine, the frequency and duration of vertigo attacks may be useful for screening for the presence of migraine in patients with MD. The main histopathology of MD is EH, and the outflow of hydrops can cause vertigo attacks. ${ }^{129}$ However, the vestibulo-thalamo-cortical pathway is always increasingly active in patients with migraine, and the degree of thalamic activation is intimately related to the frequency of migraine and vertigo attacks. ${ }^{30-32}$ We hypothesise that because EH from formation to outflow develops over a certain period of time, the vertigo cannot occur many times within a short period of time in patients with MD alone. However, in MD patients with migraine, vertigo attacks may involve both migrainous vertigo and Meniere's vertigo. In this case, not only the outflow of hydrops but also stimuli activating the thalamus can lead to vertigo attacks.

In our analysis of retinal vascular calibre between patients with MD alone and MD patients with migraine, MD patients with migraine had slightly larger CRAE than patients with MD alone. Although some studies have previously described the retinal vascular calibre in patients with migraine, the results have not always been unanimous. Gasser et al reported that the retinal vascular calibre did not differ significantly between patients with migraine and controls. ${ }^{33}$ Liew et al analysed retinal photographs of 1970 subjects aged 49 to 97 years and found that the retinal arterioles were slightly narrower in subjects with migraine than those without migraine, but the venular diameter did not differ between subjects. ${ }^{24}$ In a recent study, Unlu et al compared the retinal vessel diameters between patients with migraine and control subjects matched for age and sex and discovered that patients with $\geq 5$ migraine attacks per month had a larger retinal artery than the control group. ${ }^{34}$ Asghar et al also reported that the migraine attacks were associated with dilatation of both extracranial and intracranial vessel. ${ }^{35}$

There are several potential explanations for our findings. It is widely accepted that the activity and sensitisation of the TVS can cause headache. The active and sensitive TVS leads to the release of vasoactive neuropeptides from their perivascular endings, mainly through the ophthalmic division of the trigeminal nerve. ${ }^{36}$ It has been confirmed that the ophthalmic branch of TVS innervates the blood flow in both the retina and cochlea. ${ }^{81416}$ These vasoactive neuropeptides including substance $\mathrm{P}(\mathrm{SP})$, calcitonin gene-related peptide and neurokinin A cause vasodilation, increased cerebral blood flow, extravasation of plasma protein, release of proinflammatory factors and finally neurogenic inflammation. ${ }^{36}$ We hypothesise that when migraine attacks occur, the release of vasoactive neuropeptides can cause neurogenic inflammation and dilation of retinal blood vessels and the cochlear vasculature, which may aggravate $\mathrm{EH}$ and accelerate the outflow of EH. This may be a potential relationship between MD and migraine, and another reason why MD patients with migraine have frequent attacks. The larger artery calibre 
in MD patients with migraine compared with patients with MD alone may result from the release of vasoactive neuropeptides during headache, and the frequent release of these vasodilators may cause permanent structural changes in blood vessels.

Froster et al reviewed 3707 temporal bones with EH and found that only 165 cases could be diagnosed with MD according to the AAO-HNS 1995 criteria. ${ }^{37}$ Gurkov et al concluded that EH is necessary but not sufficient for the development of MD. ${ }^{38}$ Most animal experiments have demonstrated that MD attacks cannot be caused by a model of $\mathrm{EH}$, though they can triggered by altered vascular flow in the inner ear. ${ }^{39} 40$ Our conjecture is that migraine is a type of vascular disorder that can alter the vascular flow in the inner ear and lead to MD attacks in patients with $\mathrm{EH}$.

Our study has several limitations. First, this is a prospective, single-centre study with a limited number of patients. Second, because MD and migraine were always diagnosed based on clinical symptoms, there is a potential for recall bias. Third, patients were first treated in other hospitals, especially those who had symptoms of migraine, and may have come to our hospital for further treatment. Thus, there exists a potential for both a referral bias and a potential overestimation of the incidence of migraine in patients with MD. Finally, no single clinical characteristic or examination is specific for a diagnosis of $\mathrm{MD}$, which is based on the clinical criteria alone. Thus, there is always the potential for diagnostic error.

\section{CONCLUSIONS}

Our study indicates that a relationship exists between MD and retinal vascular calibre. Patients with MD had a slightly larger artery calibre than matched healthy controls. Increased retinal artery calibre was associated with migraine and a high frequency of vertigo attacks in patients with MD patients. We suggest that symptoms of migraine should be considered part of the standard of the classification of MD. MD was divided into two types, MD alone and MD without migraine. MD patients with migraine experienced more frequent vertigo attacks and had a larger artery calibre than patients with MD alone. More MD patients with migraine also complained of a long duration of vertigo attacks, phonophobia and visual aura. Taken together, these findings support the existence of a close pathophysiological relationship between migraine and MD attacks. Prophylactic therapy for migraine may therefore contribute to the prevention of MD attacks in MD patients with migraine. More extensive studies are required to explore the association between retinal vascular calibre and MD.

Acknowledgements We wish to thank all the patients included in this study and all the project coordinators for collecting the data.

Contributors YW built the models, analysed the data and drafted the manuscript. TD and LH were involved in reviewing the manuscript. YT is managing the measurement of retinal vascular calibres. $L Y$ was the principal investigator and managed the study. All authors read and approved the final manuscript.
Funding The authors have not declared a specific grant for this research from any funding agency in the public, commercial or not-for-profit sectors.

Competing interests None declared.

Patient consent Obtained.

Ethics approval The study was approved by the institutional review board of Peking University people'hospital, China.

Provenance and peer review Not commissioned; externally peer reviewed.

Data sharing statement The data sets used and /or analysed during the current study are available from the corresponding author on reasonable request.

Author note YW is a medical doctor studying at Peking University People's Hospital. TD is a resident doctor at Peking University People' Hospital. YT and LH are staff physicians at Peking University People's Hospital. LY is a chief physician at the Peking University People's Hospital and professor at Peking University.

Open access This is an open access article distributed in accordance with the Creative Commons Attribution Non Commercial (CC BY-NC 4.0) license, which permits others to distribute, remix, adapt, build upon this work non-commercially, and license their derivative works on different terms, provided the original work is properly cited, appropriate credit is given, any changes made indicated, and the use is non-commercial. See: http://creativecommons.org/licenses/by-nc/4.0/.

\section{REFERENCES}

1. Sajjadi H, Paparella MM. Meniere's disease. Lancet 2008;372:406-14.

2. Tyrrell JS, Whinney DJ, Ukoumunne OC, et al. Prevalence, associated factors, and comorbid conditions for Ménière's disease. Ear Hear 2014;35:e162-e169.

3. Wladislavosky-Waserman P, Facer GW, Mokri B, et al. Meniere's disease: a 30-year epidemiologic and clinical study in Rochester, Mn, 1951-1980. Laryngoscope 1984;94:1098-102.

4. Havia M, Kentala E, Pyykkö I. Prevalence of Menière's disease in general population of Southern Finland. Otolaryngol Head Neck Surg 2005;133:762-8.

5. Minor LB, Schessel DA, Carey JP. Meniere's disease. Curr Opin Neurol 2004;17:9-16.

6. Celestino D, Ralli G. Incidence of Menière's disease in Italy. Am J Otol 1991;12:135-8.

7. Foster CA, Breeze RE. The Meniere attack: an ischemia/ reperfusion disorder of inner ear sensory tissues. Med Hypotheses 2013;81:1108-15.

8. Liu YF, Xu H. The intimate relationship between vestibular migraine and meniere disease: a review of pathogenesis and presentation. Behav Neurol 2016;2016:1-8.

9. Radtke A, Lempert T, Gresty MA, et al. Migraine and Ménière's disease: is there a link? Neurology 2002;59:1700-4.

10. Cha YH, Brodsky J, Ishiyama G, et al. The relevance of migraine in patients with Meńière's disease. Acta Otolaryngol 2007;127:1241-5.

11. Neff BA, Staab JP, Eggers SD, et al. Auditory and vestibular symptoms and chronic subjective dizziness in patients with Ménière's disease, vestibular migraine, and Ménière's disease with concomitant vestibular migraine. Otol Neurotol 2012;33:1235-44.

12. Goadsby PJ, Charbit AR, Andreou AP, et al. Neurobiology of migraine. Neuroscience 2009;161:327-41.

13. Reggio E, Chisari CG, Ferrigno G, et al. Migraine causes retinal and choroidal structural changes: evaluation with ocular coherence tomography. J Neurol 2017;264:494-502.

14. Vass Z, Dai CF, Steyger PS, et al. Co-localization of the vanilloid capsaicin receptor and substance $P$ in sensory nerve fibers innervating cochlear and vertebro-basilar arteries. Neuroscience 2004;124:919-27.

15. Vass Z, Shore SE, Nuttall AL, et al. Direct evidence of trigeminal innervation of the cochlear blood vessels. Neuroscience 1998;84:559-67.

16. Friedman DI. The eye and headache. Continuum 2015;21:1109-17.

17. Heitmar R, Varma C, De P, et al. The relationship of systemic markers of renal function and vascular function with retinal blood vessel responses. Graefes Arch Clin Exp Ophthalmol 2016;254:2257-65.

18. Wagner W, Fehrmann A. Association of retinal vasculitis (Eales' disease) and Meniere-like vestibulocochlear symptoms. Eur Arch Otorhinolaryngol 2006;263:100-4.

19. Recupero SM, Mollo R, Abdolrahimzadeh S, et al. Branch retinal artery occlusion in a young woman with Ménière's disease. Ophthalmologica 1998;212:77-9. 
20. Anon. Committee on Hearing and Equilibrium guidelines for the diagnosis and evaluation of therapy in Menière's disease. American Academy of Otolaryngology-Head and Neck Foundation, Inc. Otolaryngol Head Neck Surg 1995;113:181-5.

21. Headache Classification Committee of the International Headache Society (IHS). The International Classification of Headache Disorders, 3rd edition (beta version). Cephalalgia 2013;33:629-808.

22. Hubbard LD, Brothers RJ, King WN, et al. Methods for evaluation of retinal microvascular abnormalities associated with hypertension/ sclerosis in the Atherosclerosis Risk in Communities Study. Ophthalmology 1999;106:2269-80.

23. Wang JJ, Liew G, Klein R, et al. Retinal vessel diameter and cardiovascular mortality: pooled data analysis from two older populations. Eur Heart J 2007;28:1984-92.

24. Liew G, Mitchell $P$, Wong TY, et al. Retinal vascular caliber and migraine: the Blue Mountains Eye Study. Headache 2006;46:997-1004.

25. Seth RS, Dayal D. Inner-ear involvement in primary glaucoma. Ear Nose Throat J 1978;57:355-9.

26. Shapiro A, Siglock TJ, Ritch R, et al. Lack of association between hearing loss and glaucoma. Am J Otol 1997;18:172-4.

27. Kabudwand EA, Nubel K, Gerdemann M, et al. Low-frequency masking for detection of endolymphatic hydrops in patients with glaucoma. Hear Res 1998;116:131-6.

28. Morganti LO, Salmito MC, Duarte JA, et al. Vestibular migraine: clinical and epidemiological aspects. Braz J Otorhinolaryngol 2016;82:397-402.

29. Gibson WP, Arenberg IK. Pathophysiologic theories in the etiology of Meniere's disease. Otolaryngol Clin North Am 1997;30:961-7.
30. Brandt T, Bartenstein P, Janek A, et al. Reciprocal inhibitory visualvestibular interaction. Visual motion stimulation deactivates the parieto-insular vestibular cortex. Brain 1998;121(Pt 9):1749-58.

31. Shin JH, Kim YK, Kim HJ, et al. Altered brain metabolism in vestibular migraine: comparison of interictal and ictal findings. Cephalalgia 2014;34:58-67.

32. Espinosa-Sanchez JM, Lopez-Escamez JA. New insights into pathophysiology of vestibular migraine. Front Neurol 2015;6:12

33. Gasser $P$, Meienberg $O$. Finger microcirculation in classical migraine. A video-microscopic study of nailfold capillaries. Eur Neurol 1991;31:168-71.

34. Unlu M, Sevim DG, Gultekin M, et al. Changes in retinal vessel diameters in migraine patients during attack-free period. Int $J$ Ophthalmol 2017;10:439-44.

35. Asghar MS, Hansen AE, Amin FM, et al. Evidence for a vascular factor in migraine. Ann Neurol 2011;69:635-45.

36. Pietrobon D, Moskowitz MA. Pathophysiology of migraine. Annu Rev Physiol 2013;75:365-91.

37. Foster CA, Breeze RE. Endolymphatic hydrops in Ménière's disease: cause, consequence, or epiphenomenon? Otol Neurotol 2013;34:1210-4.

38. Gürkov R, Pyykö I, Zou J, et al. What is Menière's disease? A contemporary re-evaluation of endolymphatic hydrops. J Neurol 2016;263(Suppl 1):71-81.

39. Takumida M, Akagi N, Anniko $M$. A new animal model for Ménière's disease. Acta Otolaryngol 2008;128:263-71.

40. Kimura RS. Animal models of endolymphatic hydrops. Am J Otolaryngol 1982;3:447-51. 